\title{
On minimum problems for families of functionals.
}

\author{
Masimo Furi, Mario Martelli, Alfonso Vignoli (Eirenze) (*)
}

Sommario. - Per una famiglia $\left.; I_{\lambda}\right\rangle \in \wedge \mid$ di funzionali reali definiti in uno spazio metrico $\mathrm{X}$ e dipendenti da un parametro $\lambda \in \wedge$, dove $\wedge$ è uno spazio topologico, si pone il problema di come si comporta, al variare di $\lambda$. l'insieme dei punti di minimo di $I_{\lambda}$. Lo studio di tale problema è l'oggetto del presente lavoro e ll visultato principale $\dot{e}$ dato dal Teorema 1.

In this paper we consider a family of functionals $(x, \lambda) \mapsto I(x, \lambda)$ defined on a metric space $X$, depending on a parameter $\lambda$ in a topological space $\wedge$. We investigate the dependence on $\lambda$ (in a sense to be precisely defined later) of the set of minimum points.

The main result is Theorem 1 from which some consequences (Theorem 2 and Theorem 3) and applications are derived (Corollaries 1, .., 8).

1. - We recall first some terminology.

Let $T: X \rightarrow Y$ be a continuous mapping of a metric space $(X, \rho)$ into a metric space $(Y, d)$. Consider the equation

$$
T(x)=y^{*}
$$

where $y^{*}$ is a given point of $Y$.

We recall that a point $x_{0}$ is called quasi-solution of (1) if $d\left(T\left(x_{0}\right), y^{*}\right)=$ inf $\left\{d\left(T(x), y^{*}\right) \mid x \in X\right\}$, i.e. $x_{0}$ is a minimum point for the functional $I: X \rightarrow R$ defined by $I(x)=d\left(T(x), y^{*}\right)$ (see V.K. Ivanov [1]).

Let $F: X \rightarrow X$ be a continuous mapping of a metric space $(X, d)$ into itself. The point $x_{0}$ is called an almost fixed point for $F$ if it is a minimum point for the functional $I: X \rightarrow R$ defined by $I(x)=d(F(x), x)$.

Let $I: X \rightarrow R$ be a lower bounded, lower semicontinuous real functional, defined on a complete metric space $(X, d)$. The minimum problem for the functional $I$ is said to be well-posed (well-posed in the generalized sense) if any minimining sequence $\left\{x_{n}\right\}$ is convergent (compact).

Consequently the problem of finding the quasi-solutions (almost fixed points) of $T(x)=y^{*}(F(x)=x)$ is said to be well-posed, or well-posed in the

(*) Entrata in Redazione il 3 marzo 1970 . 
generalized sense, according to whether the real functional $I: X \rightarrow R$ defined by $I(x)=d\left(T(x), y^{*}\right)(I(x)=d(F(x), x))$ is well-posed or well-posed in the generalized sense (see A. N. Tichonov [2]).

For our purposes it will be aseful the following result proved in [3]:

The minim:tm problem for the functional $I$ is well-posed (vell-posed in the generalized sense) if and only if $\varepsilon\left(\Omega_{\rho}\right) \rightarrow 0 \quad\left(\alpha\left(\Omega_{\rho}\right) \rightarrow 0\right)$ as $\rho \rightarrow 0$, where $\left.\Omega_{p}=\{x \in X \mid I(x) \leq \inf I(X)+p\}{ }^{1}\right)$.

2. - Let $X$ be a metric space (or, more generally, a topological space). By $\mathcal{S}(X)$ we denote the set of all nonempty subsets of $X$, with the upper semi-finite lopology (u.s.f. topology), i.e. the topology which has as a basis all sets of the form $\{A \in S(X), A \subset U, U$ open in $X$ \} (see T. VaN der WaLt [5]).

Let $I: X \times \wedge \rightarrow R$ be a real fanctional defined on the cartesian product $X \times \wedge$, where $X$ is a complete metric space and $\wedge$ is a topological space. In this paper by $I_{\lambda}$ we denote the partial mapping $I_{\lambda}: X \rightarrow R$ defined by $I_{\lambda}(x)=I(x, \lambda)$. We shall also use the fullowing notations:

Moreover

$$
\begin{aligned}
& \Omega_{p}(\lambda)=\left\{x \in X \mid I_{\lambda}(x) \leq \inf I_{\lambda}(X)+p\right\} ; \\
& \Omega(\lambda)=\left\{x \in X \mid I_{\lambda}(x)=\inf I_{\lambda}(X)\right\} ; \\
& B(\Omega(\lambda), \varepsilon)=\{x \in X \mid d(x, \Omega(\lambda))<\varepsilon\} .
\end{aligned}
$$

a) $I_{\lambda}(x)$ converges to $I_{\lambda_{0}}(x)$ uniformly in $X$ as $\lambda \rightarrow \lambda_{0}$ if for any $\varepsilon>0$ there exists a neighborood $N$ of $\lambda_{0}$ such that $\left|I_{\lambda}(x)-I_{\lambda_{0}}(x)\right|<\varepsilon$ for any $x \in X, \lambda \in N$;

b) $\Omega_{p}(\lambda) \stackrel{\text { u.s.f. }}{\rightarrow} \Omega\left(\lambda_{0}\right)$ as $(\lambda, \rho) \rightarrow\left(\lambda_{0}, 0\right)$ if for any open set $U$ containing $\Omega\left(\lambda_{0}\right)$, there exists a neighborood $N$ of $\lambda_{0}$ and $\delta>0$ such that $\Omega_{f}(\lambda) \subset U$ for any $\lambda \in N, 0<\rho<\delta$.

THeorem 1. - Let $X$ be a comptete metric space and $\wedge$ a topological space. Let $I: X \times \wedge \rightarrow R$ be a real functional such that $I_{\lambda}$ is lower bounded for any $\lambda \in \wedge$. Let $\lambda_{0}$ be a given point of $\wedge$. Assume that

i) $I_{i, 0}$ is lower semicontinuous;

ii) $I_{\lambda}(x)$ converges to $I_{\lambda .0}(x)$ uniformly in $X$ as $\lambda \rightarrow \lambda_{0}$;

iii) the minimum problem for $I_{\lambda_{0}}$ is well-posed in the generalized sense.

(1) Let $A$ be a subset of a metric space $(X, d)$. By $\delta(A)$ and $x(A)$ we denote the diameter and the Kuratowski number of $A$, respectively (see [4]). 


\section{Then:}

a) $\Omega\left(\lambda_{0}\right)$ is nonempty and compact;

b) $\Omega_{p}(\lambda) \stackrel{\text { n.s.8. }}{\rightarrow} \Omega\left(\lambda_{0}\right)$ as $(\lambda, \rho) \rightarrow\left(\lambda_{0}, 0\right)$.

Proof. - The assertion a) follows immediately from the definition of well-posed problem in the generalized sense.

In order to prove the assertion b) let $U$ be an open set containing $\Omega\left(\lambda_{0}\right)$. Since $\Omega\left(\lambda_{0}\right)$ is compact there exists $\varepsilon>0$ such that $B\left(\Omega\left(\lambda_{0}\right), \varepsilon\right) \subset U$. But from iii) it follows that $x\left(\Omega_{\rho}\left(\lambda_{0}\right)\right) \rightarrow 0$ as $\rho \rightarrow 0$ (see [3]); hence there exists $\delta>0$ such that $\Omega_{38}\left(\lambda_{0}\right) \subset B\left(\Omega\left(\lambda_{0}\right)\right.$, $\left.\varepsilon\right)$ (see [4]). By ii) there exists a neighborood $N$ of $\lambda_{0}$ such that $\lambda \in N \Rightarrow\left|I_{\lambda}(x)-I_{\lambda_{0}}(x)\right|<\delta$ independently of $x \in X$. It remains to prove that if $0<\rho<\delta$ and $\lambda \in N$, then $\Omega_{\rho}(\lambda) \subset B\left(\Omega\left(\lambda_{0}\right), \varepsilon\right) \subset U$, i.e. if $x \in \Omega_{d}(\lambda)$ we have $d\left(x, \Omega\left(\lambda_{0}\right)\right)<\varepsilon$.

Obviously

$I_{\lambda_{0}}(x)-\inf I_{\lambda_{0}}(X) \leq\left|I_{\lambda_{0}}(x)-I_{\lambda}(x)\right|+I_{\lambda}(x)-\inf I_{\lambda}(X)+\mid \inf I_{\lambda}\left(X\left(-\inf I_{\lambda_{0}}(X) \mid\right.\right.$.

Since $x \in \Omega_{\rho}(\lambda)$, it follows that

$$
I_{\lambda}(x) \leq \inf I_{\lambda}(X)+\rho, \quad \text { i.e. } I_{\lambda}(x)-\inf I_{\lambda}(X)<\delta
$$

Moreover, it is easy to see that for any $\lambda \in N$ we have

$$
\left|\inf I_{\lambda}(X)-\inf I_{\lambda .0}(X)\right|<\delta, \quad \text { since } \quad\left|I_{\lambda}(x)-I_{\lambda .0}(x)\right|<\delta
$$

for any $x \in X, \lambda \in N$. Hence, if $x \in \Omega_{\rho}(\lambda), I_{\gamma_{0}}(x)-\inf I_{\lambda_{0}}(X)<3 \delta$.

Then $d\left(x, \Omega\left(\lambda_{0}\right)\right)<\varepsilon$.

As a particular case of Theorem 1 we have the following result proved in [6].

COROLLARX 1. - Let $\left(T_{n}\right)$ be a sequence of mappings of a complete netric space $(X, d)$ into itself, which converges uniformly to a continuous mapping T. Put $\Omega_{p}=\{x \in X \mid d(T(x), x) \leq p)$. If $\alpha\left(\Omega_{f}\right) \rightarrow 0$ as $p \rightarrow 0$, then any sequence $\left\{x_{n}\right\}$, such that $d\left(T_{n}\left(x_{n}\right), x_{n}\right) \rightarrow 0$, is compact and all its limit points are fixed for $T$.

Proof. - Let $\wedge=\left\{0,1, \frac{1}{2}, \ldots, \frac{1}{n}, \ldots\right\}$ with the relative topology as a subset of $R$. Put

$$
I_{\lambda}(x)= \begin{cases}d(T(x), x) & \text { if } \quad \lambda=0 \\ d\left(T_{n}(x), x\right) & \text { if } \quad \lambda=1 / n\end{cases}
$$

Clearly, since $\alpha\left(\Omega_{\beta}\right) \rightarrow 0$ as $\rho \rightarrow 0$, the minimum problem for the functional $I_{0}$ is well-posed in the generalized sense and, since 


$$
\left|d(x, T(x))-d\left(x, T_{n}(x)\right)\right| \leq d\left(T(x), T_{n}(x)\right),
$$

$I_{\lambda}(x)$ converges to $I_{0}(x)$ uniformly in $X$ as $\lambda \rightarrow 0$. Obviously $x_{n} \in \Omega_{\rho_{n}}(1 / n)$, where $p_{n}=d\left(T_{n}\left(x_{n}\right), x_{n}\right) \rightarrow 0$. Then, by Theorem $1, \Omega(0)$ is nonempty and compact and $d\left(x_{n}, \Omega(0)\right) \rightarrow 0$. Therefore $\left\{x_{n}\right\}$ is compact and all its limit points belong to $\Omega(0)$.

3. - The following theorem is a consequenee of Theorem 1 and it is less general, but more usefnl for some applications.

Theorem 2. - Let $I: X \times \wedge \rightarrow R$ be a real functional, where $X$ is a complete metric space and $\wedge$ is a topological space. Let $1_{\lambda}$ be a lower bounded, lower semicontinuous for all $\lambda \in \wedge$. If for any $\lambda_{0} \in \wedge, I_{2}(x)$ converges to $I_{\lambda_{0}}(x)$ unitormly in $X$ as $\lambda \rightarrow 0$ and the minimum problem for $I_{\lambda_{0}}$ is wel-lposed in the generalized sense, then the set-ralued mapping $\varphi: \wedge \rightarrow \varsigma(X)$, defined by $\varphi(\lambda)=\Omega(\lambda)$, is upper semicontinuous, i.e. is continuous in the u.s.f. topology of $\mathfrak{S}(X)$.

Proof. - Since the minimum problem for the functional $I_{\lambda}$ is well-posed in the generalized sense for any $\lambda \in \Lambda$ then the sets $\Omega(\lambda), \lambda \in \Lambda$, are nonempty and compact. On the other hand $\Omega(\lambda) \subset \Omega_{\rho}(\lambda)$ for any $\lambda$ and $\rho>0$. Hence, by Theorem 1 , the set-valued mapping $\varphi$ is upper semicontinuous.

A continuous mapping $T: D \rightarrow X$, defined on a subset $D$ of a metric space $X$, is called densifying if $\alpha(T(A))<\alpha(A)$ for any bounded set $A \subset D$, $\alpha(A)>0$ (see [7]).

It is easy to see that contractive mappings and completely continuous mappings are densifying, as well as sum of these two types of mappings in Banach spaces.

Corollant 2. - Let $D$ be a closed and bounded set of a Banach space $X$. Let $T: D \rightarrow X$ be a densifying mapping and consider the equation

$$
x+T(x)=y,
$$

where $y$ is a given point of $X$. Let $E=\{x+T(x) \mid x \in D\}$. Then the set-valued mapping $\Gamma: E \rightarrow \mathfrak{S}(D)$, defined by $\Gamma(y)=\{x \in X \mid x+T(x)=y\}$ is upper seni. continuous, ie. is continuous in the u.s.f. topology of $\$(D)$. follows

Proof. - Put $F_{y}(x)=y-T(x)$. Then the equation (1) can be written as

$$
x=F_{y}(x),
$$

where $F_{y}$ is a densifying mapping for all $y \in E$. Hence, for any $y \in E$, the minimum problem for the lower bounded, continuous functional $I_{y}: D \rightarrow R$, 
defined by $I_{y}(x)=\left\|x-F_{y}(x)\right\|$ is well-posed in the generalized sense (see [8]).

Olearly $I_{y}(x)$ converges to $I_{y_{0}}(x)$ uniformly in $D$ as $y \rightarrow y_{0}$, for any $y_{0} \in E$. The assertion follows by Theorem 2.

Corollary 3. - Let $f: X \rightarrow Y$ be a continuous mapping of a compact metric space $(X, F)$ onto a metric space $(Y . d)$. Then the set-valued mapping $\bar{f}: y \rightarrow \mathfrak{S}(X)$ defined by $\bar{f}(y)=\{x \in X|f(x)=y|$ is upper semicontinuous.

Procf. - Put $I_{y}(x)=d(f(x), y)$ and let $y_{0}$ be a given point. We have

$$
\left|d(f(x), y)-d\left(f(x), y_{0}\right)\right| \leq d\left(y, y_{0}\right)
$$

Hence $I_{y}(x)$ converges to $I_{y_{0}}(x)$ uniformly in $X$ as $y \rightarrow y_{0}$. Moreover, by the compactness of $X$, the minimum problem for the functional $I_{y}$ is wellposed in the generalized sense for any $y \in Y$. The assertion follows by Theorem 2.

Corollary 3 can be extended as follows.

Corollary 4. - Let $f: X \rightarrow Y$ be a continuous mapping of a complete metric space $(X, f)$ onto a metric space $(Y, d)$. Put

$$
\Omega_{\varepsilon}(y)=\{x \in X \mid d(f(x), y)<\varepsilon\}
$$

i.e. $\Omega_{\varepsilon}(y)$ is the reverse image of the set $B(y, \varepsilon)$. If, for any $y \in Y, \alpha\left(\Omega_{\varepsilon}(y)\right) \rightarrow 0$ as $\rho \rightarrow 0$, then the set-valued mapping $\bar{f}: Y \rightarrow S(X)$ defined by $\bar{f}(y)=|x \in X|$ $f(x)=y\}$ is upper semicontinuous.

Proof. - Put $I_{y}(x)=d(f(x), y)$. Since $\alpha\left(\Omega_{\varepsilon}(y)\right) \rightarrow 0$ as $\varepsilon \rightarrow 0$, for any $y \in Y$ the minimum problem for the functional $I_{y}$ is well-posed in the generalized sense (see [3]). Moreover, as in Corollary $2, I_{y}(x)$ converges to $I_{y_{0}}(x)$ uniformly in $X$ as $y \rightarrow y_{0}$, for any $y_{0} \in Y$. The conclusion follows again by Theorem 2 .

Let $\left(R^{n},|\cdot|\right)$ be the $n$-dimensional, real euclidean space and let $C_{n}[a, b]$ be the Banach space of all continuous mappings $\varphi:[a, b] \rightarrow R^{n}$ with norm

$$
\|\varphi\|=\max \{|\varphi(t)|: t \in[a, b]\}
$$

Consider the differential equation

$$
\dot{x}=f(t, x)
$$

where $f:[a, b] \times R^{n} \rightarrow R^{n}$ is bounded and continuous.

Let $D \subset C_{n}[a, b]$ be the set of all solutions of the differential equation (3). Then, as a consequence of Corollary 4, we have 
Coroluary 5. - The mapping $\sigma: R^{n} \rightarrow \mathcal{S}(D)$ defined by $\sigma(\lambda)=\{\varphi \in D \mid \psi(a)=\lambda\}$ is continuous in the u.s.f. topology of $\$(D)$.

Conollary 6. - Let $T: Q \times \wedge \rightarrow Q$ be a continuous mapping, where $Q$ is a compact and convex set of a Banach space $X$ and $\wedge$ is a topological space. If, for any $\lambda_{0} \in \Lambda, T_{\lambda}(x)$ concerges to $T_{\gamma_{0}(x)}$ uniformly in $Q$ as $\lambda \rightarrow \lambda_{0}$, then the set-valued mapping $\vartheta: \wedge \rightarrow S(Q)$ defined by $\varphi(\lambda)=\left\{x \in Q \mid T_{\lambda}(x)=x\right\}$ is upper semicontinuous.

Proof. - Put $I_{\lambda}(x)=\left\|T_{\lambda}(x)-x\right\|$ and let $\lambda_{0} \in \wedge$ be a given point. Clearly we have

$$
\left|I_{\lambda}(x)-I_{\lambda_{0}}(x)\right|=\mid\left\|T_{\lambda}(x)-x\right\|-\left\|T_{\lambda_{0}}(x)-x\right\| \leq\left\|T_{\lambda}(x)-T_{\lambda_{0}}(x)\right\| .
$$

Hence $I_{\lambda}(x)$ converges to $I_{2.0}(x)$ uniformly in $X$ as $\lambda \rightarrow \lambda_{0}$. But, for any $\lambda \in \wedge, T_{\lambda}$ is a continuous mapping of $Q$ into itself; by the ScHaUder fixed point theorem, there exists $x_{\lambda} \in Q$ such that $T_{\lambda}\left(x_{\lambda}\right)=x_{\lambda}$. Then $\Omega(\lambda) \neq \emptyset$.

Therefore, by the compactness of $Q$, the minimum problem for the functional $I_{\lambda}$ is well-posed in the generalized sense for any $\lambda \in \wedge$. Hence $\varphi$ is continuous in the u.s.f. topology of $\$(Q)$ by Theorem 2.

4. - As a consequence of Theorem 2 we have

Theorem 3. - Let $I: X \times \wedge \rightarrow R$ be a real functional, where $X$ is a complete metric space and $\wedge$ is a topological space. Let, for all $\lambda \in \wedge, I_{\gamma}$ be lower bounded, lower semicontinuous. If, for any $\lambda_{0} \in \wedge, I_{\lambda}(x)$ converges to $I_{\lambda_{0}}(x)$ uniformly in $X$ as $\lambda \rightarrow \lambda_{0}$ and the minimum problem for $I_{\lambda_{0}}$ is wellposed, then the mapping $\psi: \wedge \rightarrow X$, where $\psi(\lambda)$ is the unique minimum point of $I_{\lambda}$, is continuous.

Proof. - The uniqueness of the minimum point for $I_{\lambda}$ follows immedia. tely from the definition of well-posed problem. Since the injection $i: X \rightarrow \mathfrak{S}(X)$ defined by $i(x)=\{x\}$ is an imbedding the assertion follows by Theorem 2 .

Corollary 7. - Let $T: X \times \wedge \rightarrow X$ be a continuous mapping, where $X$ is a complete metric space and $\wedge$ is a topological space. If for any $\lambda_{0} \in \wedge$, $T_{\lambda_{0}}$ is a contractive mapping and $T_{\lambda_{1}}(x)$ converges to $T_{\lambda_{0}}(x)$ uniformly in $X$ as $\lambda \rightarrow \lambda_{0}$, then there exists a unique mapping $\psi: \Lambda \rightarrow X$, such that $\psi(\lambda)=T(\psi(\lambda), \lambda)$ and $\psi$ is continuous.

Proof. - The uniqueness follows immediately from Banach contraction principle. Put $I_{\lambda}(x)=d\left(x, T_{\lambda}(x)\right)$. It is easy to see that $\delta\left(\Omega_{0}(\lambda)\right) \leq 2 \rho /\left(1-k_{\lambda}\right)$, where $k_{\lambda}<1$ is the Lipschitz constant of $T_{\lambda}$. Therefore the minimum problem for $I_{\lambda}$ is well posed for every $\lambda \in \wedge$ (see [3]) and so, by Theorem 3 , $\psi$ is continuous. 
Let $T: X \rightarrow X$ be a continuous mapping of a complete metric space $(X, d)$ into itself, with a unique fixed point $\xi \in X$. We recall that the uniqueness of $\xi$ is said to be strong (P. ZECOA [9]) if for every $\varepsilon>0$ there exists $\delta>0$ such that

$$
d(x, T(x))<\delta \Rightarrow d(x, \xi)<\varepsilon
$$

Corollary 8. (S. Dragoni - [10]) Let $T: X \times \wedge \rightarrow X$ be a continuous mapping, where $X$ is a complete metric space and $\wedge$ is a metric space. For any $\lambda_{0} \in \wedge$ suppose that $T_{\lambda}(x)$ converges to $T_{\lambda_{0}}(x)$ uniformly in $X$. If $T_{\lambda}$ has a unique fixed point $\xi_{\lambda}$ for any $\lambda$, and the uniqueness of $\xi_{\lambda}$ is strong, then the mapping $\psi: \lambda \rightarrow \xi_{\lambda}$ is continuous.

Proof. - It is easy to see that the strong uniqueness implies that the minimum problem for the functional $I_{\lambda}: X \rightarrow R$ defined by $I_{\lambda}(x)=d\left(x, T_{\lambda}(x)\right)$ is well posed for any $\lambda \in \wedge$. Then, by Theorem 3 , the mapping $\psi$ is continuous.

\section{REFERENCES}

[1] V.K. Ivanov, About non-comectly posed problems, Matematiceskij Sbornik, Vol. 61, No. 2, (1968), (in russian).

[2] A. N. 'ICHonov, On the stability of functional optimization problem, U.R.S.S., Computational Mathematies and Mathematical Physies, Vol. 16, No. 4, (1966).

[3] M. Fur and A. VrgyoLr, About well-posed optimization problems for functional in metric spaces, Journal of Optimization Theory and Applications, Vol. 5, No. 3, (1970).

[4] C. Koratowsi, Topologie, "Monografie Matematyozne, tom. 20, Warszawa, (1958).

[5] T. VAN Der WALT, Fixed and almost fixed points, Mathematisch Centrum Amsterdam, Mathematical Centre Tracts, 1, (1967).

[6] M. EURI e M. MarTaL,I, Successioni di trasformazioni in uno spazio metrico e punti fissi, Rendiconti Accademia Nazionalo Lincei, Vol. XLVII, No. 1-2, (1969).

[7] M. Fur and A. Vigyorr. A fixed point theorem in complete metric spaces, Bollettino Unione Matematica Italiana, Serie IV, No. 4.5, (1969).

[8] - - and - - Fixed points for densifying mappings, Rendiconti Accademia Nazionale Lincei, Vol. XLVIT, No. 6, (1969).

[9] P. ZECCA, Un teorema di esistenza per l'elemento unito di un sistema di trasformazioni funzionali, Rend. Ace. Se. Fis. Mat. Napoli, Vol. XXXV, (1968); pp. 27.46.

[10] C. Scorza Dragon, Elenenti uniti di trasformazioni funzionali e teoremi di dipen. denza contimua, Atti Istituto Veneto di Scienze, Lettere e Arti, Vol. XCIX, (1989), pp. $147-151$. 